\title{
Erosión hídrica en suelos de laderas cultivadas con café sin sombra, en Copán Honduras
}

Juan Manuel López Guevara ${ }^{1}$

\section{RESUMEN}

Se analizaron efectos de la erosión hídrica en suelos de laderas cultivados con café sin sombra en una zona del departamento de Copán Honduras, durante la época lluviosa de 2016, por medio de la estimación de las variaciones en la pérdida del suelo y en el escurrimiento superficial. Se planteó un diseño completamente al azar, cuyas unidades experimentales fueron constituidas por parcelas de escorrentía y erosión instaladas en áreas con $46 \%$ promedio de pendiente, cultivadas con café en tres diferentes rangos de edades. Los resultados del análisis de varianza muestran diferencias estadísticas que indican que la reducción de la escorrentía y de las pérdidas de suelo se deben a cambios en la densidad del follaje del cultivo del café a medida que va creciendo. Los análisis de correlación y regresión entre la precipitación, la escorrentía, y entre ésta y la pérdida de suelo muestran que existe una asociación positiva media en ambos casos. En todos los casos, la diferencia en los volúmenes de escorrentía y las pérdidas por erosión entre la cobertura boscosa y las diferentes edades es significativa, demostrando la capacidad del follaje para interceptar y amortiguar la energía cinética de las gotas de lluvia que generan el arrastre de las partículas de suelo, y el potencial de absorción y almacenamiento de la capa de material orgánico que cubre el suelo y del tejido radicular para influir en la circulación del agua en el suelo.

Palabras clave: : Erosión hídrica, escurrimiento superficial, cultivos de café

\footnotetext{
${ }^{1}$ Profesor en el Centro Universitario Regional de Occidente (CUROC) de la UNAH: lopezgjm@gmail.com
} 


\section{ABSTRACT}

The study assesses the effects of water erosion on slope soils cultivated with no shaded coffee in the protected area named Cocoyagua, at the western department of Copan, Honduras, during the 2016 rainy season, through the estimation of variations of soil loss, of the surface runoff and of the soil nutrient content. a completely randomized design was outlined, whose treatments were coffee crop plots of three different age's ranges, plus the comparison group, it is to say, and forest plots adjacent to coffee plantations. Experimental units were composed by runoff and erosion plots, installed at coffee cultivated and at the woods plots, with average slope of $46 \%$. Analysis of Variance (ANOVA) results show statistical differences indicating that runoff and soil loss reduction are due to foliar density changes of the coffee plantation according to its growth process, although post hoc tests don't show the same coincidence on all results. Correlation and regression analysis between precipitation and runoff and between this one and the soil loss indicate an intermediate positive association in both cases. In all cases, difference in runoff and soil erosion loss between woods protection and the different coffee ages is significant, demonstrating foliage capacity for intercepting and absorbing raindrops kinetic energy that cause soil particles dragging, and the potential of absorption and storage of the organic material covering the soil and of the root tissue for influencing on the soil water circulation

Keywords: Water erosion, surface runoff, coffee crops 


\section{INTRODUCCIÓN}

La erosión hídrica de los suelos es considerada uno de los más importantes problemas ambientales en el mundo (Konz, Baenninger, Konz, Nearing y Alewell, 2010), tan grave como el calentamiento global y la pérdida de biodiversidad, y el principal que aqueja la agricultura (Hincapié y Ramírez, 2010), cuyo control es uno de los principales temas de la agenda ambiental mundial (Ananda y Herat, 2003, citados por Cisneros, et al, 2012; Lobos, ed., 2013). Las repercusiones se manifiestan en pérdida de la productividad y degradación de los suelos (Hincapié y Salazar, 2011), y según Zamora, (2011), abandono del campo y pobreza extrema.

Los suelos de laderas de la región occidental, particularmente aquéllos cuyo uso es destinado a la producción de café, son vulnerables ante los impactos del cambio climático, estimándose, como en otros suelos de laderas del país, que "la reducción de los niveles de humedad del suelo, propiciarían la erosión y eventualmente el inicio de procesos de desertización en los suelos de laderas" (Secretaría de Recursos Naturales y Ambiente [SERNA], 2010, p. 20). La erosión se ha visto acelerada por las prácticas de agricultura migratoria, la ganadería extensiva, los incendios forestales y los aprovechamientos ilegales de productos derivados del bosque. Otras situaciones que se presentan en los sistemas de producción se integran en la problemática, como la poca diversificación de cultivos, la ausencia de prácticas de conservación y mejoramiento de los suelos, las condiciones inseguras de tenencia de la tierra y la poca utilización de tecnologías apropiadas en los cultivos.

Los estudios relacionados con los suelos, particularmente enfocados al análisis de los procesos erosivos, son escasos en la región occidental del país. Es por ello, que la ejecución de investigaciones que conduzcan a propuestas que promuevan el manejo racional del recurso suelo, particularmente en aquellas áreas dedicadas a la producción cafetalera, que es el rubro agrícola de mayor importancia para la región, se hacen necesarias.

Se espera que la información generada contribuya a crear conciencia en los productores e instituciones acerca de los impactos de la pérdida de suelos en la productividad y en la salud de los ecosistemas y al diseño de estrategias integradas de manejo de los suelos en la zona, minimizando la pérdida de suelos, aprovechando los nutrientes que se encuentran en el suelo y conservando los recursos naturales.

Se procura validar la aplicabilidad de una técnica conocida y utilizada en forma recu- 
rrente en diferentes lugares para determinación de la erosión del suelo. La técnica de las parcelas de escorrentía y erosión es indicada cuando la finalidad es demostrar hechos conocidos, en estudios comparativos o para obtener datos para construir 0 validar un modelo o ecuación destinado a predecir la escorrentía o la pérdida de suelo (Hudson, 1997). Existen registros del desarrollo incipiente de la técnica de las parcelas de escorrentía desde la segunda década del siglo XX (Hayward, 1968). Desde entonces, se han conocido experiencias de uso del método en diferentes partes del mundo: en Suiza (Konz et al., 2010), Chile (Vega, 2008), Ecuador (Chela, Monar, Valverde y Cruz, 2008), España (de Alba, Benito y Pérez, 2002), Guatemala (Santos, 2010) y Costa Rica (Garzón, Faustino, Ferreira y Kass, 1993), entre otros. Se han desarrollado manuales y lineamientos metodológicos para el diseño y las mediciones (Perret, 1993, Felipe-Morales, 1996; Hudson, 1997; Programa para la Agricultura Sostenible en Laderas de América Central [PASOLAC], 2005; Da Silva, Vaquero y Tejada, 2006).

\section{METODOLOGIA}

Diseño: La investigación presenta un diseño experimental, que se ejecutó a nivel de campo, en el ambiente natural en el que ocurren los fenómenos que generan la erosión del suelo. Los tratamientos seleccionados fueron: a) cultivo de café sin sombra en suelos de ladera, en tres diferentes rangos de edades: $\approx 1$ año; $\approx 2$ años; $\approx 3$ años; b) cobertura forestal, que constituye el grupo de control del experimento.

Se utilizó el diseño completamente al azar (DCA). Se plantearon tres repeticiones por cada tratamiento, más el grupo de control, para un total de 12 unidades experimentales. Cada unidad experimental (parcela de escorrentía) se instaló en un área promedio de $42 \mathrm{~m} 2$; las que se instalaron en el bosque, en un área de $55 \mathrm{~m} 2$. Las parcelas se instalaron en terrenos con rangos de pendientes ente 40 y $50 \%$.

Población sobre la que se ha hecho el estudio: El universo está constituido por áreas de terreno, que según Pennock, (2004) deben compartir algunas características comunes. Criterios como los planteados por Da Silva, Vaquero y Tejada (2006), fueron aplicados para la selección de los sitios de medición: a) manejo agronómico similar en todos los lotes; b) microrrelieve uniforme de la pendiente del terreno; c) textura similar del suelo de las parcelas; d) el tamaño del área cultivada debe permitir la instalación de las parcelas y sus equipos de medición de escorrentía; e) los sitios de medición deben ser de fácil acceso y permitir el monitoreo de los mismos. 
Las unidades experimentales corresponden a las parcelas de escorrentía y erosión, que se instalaron dentro de la zona de estudio; éstas son esencialmente instrumentos evaluación a nivel de campo que permiten medir la producción de escorrentía y sedimentos en "una superficie de terreno delimitada, de la que se conoce con detalle sus características edáficas, topográficas y morfológicas, y aquellas relativas al estado de la vegetación, recubrimiento del suelo, prácticas de manejo, etc." (De Alba, Benito y Pérez, 2002, p. 45).

Entorno: El área de estudio se localiza en el departamento de Copán, en la región occidental de Honduras, dentro de la zona conocida como Cocoyagua, declarada como Zona de Vocación Forestal. Es una zona montañosa y de relieve irregular, con un amplio sistema de drenaje. La altitud se encuentra entre 900 y $1500 \mathrm{msnm}$. La precipitación ocurre entre 1300 y $1500 \mathrm{~mm}$ de agua por año, la temperatura varía entre 20 y $30^{\circ} \mathrm{C}$ (Consejo Intermunicipal Higuito, 2006). Los tipos de uso de suelo son el bosque latifoliado, el cultivo de café, de granos básicos y la ganadería extensiva. La vegetación muestra fuertes influencias antropogénicas debido a acciones de tala - roza y quema realizadas en las últimas décadas para el establecimiento y manejo de cultivos.

Intervenciones: Las parcelas de escorrentía y erosión están constituidas por un campo experimental, "de forma rectangular ubicado en el sentido de la pendiente del terreno" (Felipe-Morales, 1996, p. 13). Se construyeron de láminas metálicas incrustadas en el suelo unos $20 \mathrm{~cm}$, y sobresaliendo sobre la superficie otros $20 \mathrm{~cm}$. El sistema receptor, ubicado en el lado inferior del campo, se conformó por un canal colector, un canal conductor y tanques receptores cilíndricos, integrados por un tanque principal y un auxiliar.

La toma de datos de los volúmenes de escurrimiento superficial se realizó entre los meses de mayo y septiembre, cada vez que se registró un evento de lluvia, midiendo la profundidad de agua escurrida en el tanque, para luego determinar el volumen en m3/ha. Para medir las pérdidas de suelo se agitó el tanque, se tomó una muestra de un litro, se filtró y se determinó el peso de los sólidos secados a una temperatura aproximada de $60^{\circ} \mathrm{C}$. Este valor se multiplicó por el volumen colectado en el tanque para calcular la pérdida de suelo, que luego se expresó en ton/ha. Se determinó la variación de nutrientes, tomando muestras del suelo erosionado a $20 \mathrm{~cm}$ de profundidad en cada parcela de escurrimiento al inicio, a la mitad y al final del periodo de estudio, que fueron enviadas a un laboratorio para determinar las concentraciones de nutrientes en cada parcela. 


\section{ANÁLISIS DE DATOS}

El estudio mide las variaciones del escurrimiento superficial, el suelo erosionado y los nutrientes perdidos en cada una de las parcelas de escorrentía y entre los tratamientos. La organización y el procesamiento de los datos se realizó utilizando hojas de cálculo de Excel y el programa estadístico SPSS.

El análisis se realizó por medio de medidas de estadística descriptiva de los lotes de escorrentía y los tratamientos. El análisis estadístico inferencial se realizó por medio del Análisis de Varianza (ANOVA) para determinar las diferencias estadísticas de los volúmenes de agua escurrida, de las cantidades de suelo erosionado y de las pérdidas de nutrientes entre tratamientos, analizando las variaciones con niveles de significancia $a=0.05$ y $a=0.01$. Posteriormente, se realizaron pruebas post hoc de las medias (la prueba de rango de la diferencia altamente significativa [DHS] de Tukey), para determinar las diferencias entre las medias en forma individual entre cada uno de los tratamientos.

Se llevó a cabo un análisis para determinar el grado de asociación entre los factores estudiados por medio del coeficiente de correlación $r$ de Pearson y el análisis de regresión lineal. Los análisis de correlación se sustentaron con hipótesis sometidas a prueba. Se realizaron análisis de significancia por medio de la distribución muestral $t$ y la prueba de razón F. Las correlaciones que se analizaron fueron: volumen de precipitación y de escorrentía, y volumen de escorrentía y el peso de suelo erosionado.

\section{RESULTADOS}

\section{Variaciones en los volúmenes de escurrimiento superficial}

La precipitación acumulada durante el período de estudio alcanzó $750 \mathrm{~mm}$, registrándose precipitaciones máximas de $42 \mathrm{~mm}$ y medias de $18.29 \mathrm{~mm}$. Se presentaron valores elevados a mediados del mes de junio, a finales de agosto y durante el mes de septiembre, mientras que los volúmenes más bajos se observaron de mediados de julio a mediados de agosto, período que corresponde a la ocurrencia de la canícula en la zona.

Los resultados sugieren que el escurrimiento del agua se va reduciendo a medida 
que se incrementa el área foliar en la plantación de café, y es considerablemente menor en las parcelas de bosque en las cuales se presenta una mayor cobertura foliar que intercepta el agua precipitada, además de la presencia de una mayor cantidad de material orgánico que absorbe el agua que se deposita sobre la superficie del suelo.

En general, las cantidades de agua escurrida son bajas (menos de $20 \%$ ) en relación a las cantidades de agua interceptada e infiltrada. El coeficiente de escurrimiento encontrado en cada uno de los tratamientos disminuye a medida que se incrementa la edad del cultivo, desde 0.22 en el tratamiento $1(\approx 1$ año), hasta 0.13 en el $3(\approx 3$ años). El grupo de comparación presentó un coeficiente de 0.05 . Esa disminución expresa una disminución en la cantidad de la escorrentía superficial en relación con los volúmenes de precipitación caída.

Se utilizó un ANOVA para probar las diferencias entre las medias del escurrimiento superficial entre cada uno de los tratamientos. El estadístico $\mathrm{F}$ de la prueba calculado fue 30.78, mayor que el tabulado (3.94), a un nivel de significancia $a=0.01$ (Little y Hills, 1989), por lo tanto, se rechaza la hipótesis nula y se acepta la hipótesis alternativa en el nivel de confianza de $99 \%$ (cuadro 1). Se concluye que existen diferencias estadísticas altamente significativas entre las medias de los tratamientos para rechazar la hipótesis nula, lo que indica que la variabilidad entre las edades no se debe al azar sino al efecto de los cambios en el follaje entre ellas.

\section{Cuadro 1. Resumen de las fuentes de variación (tratamientos incluyendo el control).}

\begin{tabular}{|c|c|c|c|c|c|c|}
\hline $\begin{array}{c}\text { Fuente de } \\
\text { variación }\end{array}$ & $\begin{array}{c}\text { Suma de } \\
\text { cuadrados }\end{array}$ & $\begin{array}{c}\text { Grados } \\
\text { de } \\
\text { libertad }\end{array}$ & $\begin{array}{c}\text { Varianza de } \\
\text { los cuadrados } \\
\text { medios }\end{array}$ & \multicolumn{2}{|c|}{$\begin{array}{c}\text { Valores tabulados } \\
\text { de la razón } F\end{array}$} & $\begin{array}{c}\text { F calculado }= \\
\text { CME/CMD }\end{array}$ \\
\hline $\begin{array}{c}\text { Entre tratamientos } \\
\text { (SCE) }\end{array}$ & $8,179.25$ & 3 & $2,726.42$ & $a=0.05$ & $\mathrm{~F}=2.68$ & 30.78 \\
\hline $\begin{array}{c}\text { Dentro de } \\
\text { tratamientos (SCD) }\end{array}$ & $12,044.64$ & 136 & 88.56 & $\mathrm{a}=0.01$ & $\mathrm{~F}=3.94$ & \\
\hline Total (SCT) & $20,223.89$ & 139 & 145.50 & & & \\
\hline
\end{tabular}

Fuente: Elaboración propia. 
Habiendo obtenido diferencias significativas entre los tratamientos, se realizaron pruebas de rango post ANOVA para determinar las diferencias particulares entre cada uno de ellos. La prueba de rango DHS de Tukey, con un nivel de confianza de 95\%, dio un valor de 5.77 , por consiguiente, una diferencia de por lo menos $5.77 \mathrm{~m} 3 / \mathrm{ha}$ entre cualquier par de medias resulta estadísticamente significativa.

Las diferencias en el volumen de escorrentía no son significativas entre las edades de $\approx 1$ año $\mathrm{y} \approx 2$ años, ni entre $\approx 2 \mathrm{y} \approx 3$ años, probablemente por ser períodos relativamente cortos en los que el follaje desarrollado no produce la suficiente cobertura para reducir de forma significativa la escorrentía. Son significativamente diferentes entre las edades de $\approx 1$ y $\approx 3$ años, dada una mayor amplitud de tiempo en la que se pueden producir cambios en el follaje de las plantas que se manifiesten en una reducción significativa de la escorrentía (Gráfico 1).

\section{Gráfico 1. Diagrama de árbol de las diferencias entre medias para las compara- ciones de la prueba de rango DHS del escurrimiento superficial.}

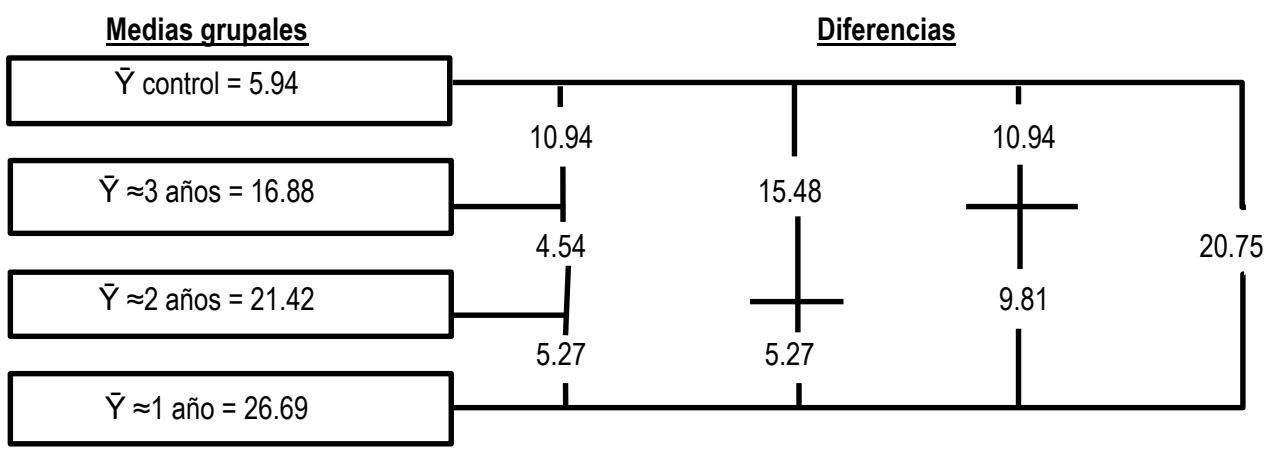

Fuente: Elaboración propia.

El volumen de escorrentía es significativamente diferente entre el grupo de control y cada uno de los tratamientos. La cobertura boscosa presenta una marcada superioridad en la intercepción de las gotas de lluvia y en la absorción de agua por el material orgánico depositado en el suelo y por el sistema de raíces de las plantas.

\section{Variaciones en la cantidad de suelo erosionado}

La cuantificación de los datos de pérdida de los suelos por erosión en cada uno de los lotes proporciona promedios hasta de 25 ton/ha. Se encontraron pérdidas promedio de más de 18 Ton/ha en los lotes de $\approx 1$ año, que se reducen hasta cerca de 6 Ton/ha en los lotes de $\approx 3$ años y a menos de 1 Ton/ha en el área boscosa. 
Las diferencias entre las medias de las pérdidas de suelo por erosión en cada uno de los tratamientos se analizaron por medio de un ANOVA, calculándose el estadístico $F$ de la prueba, que dio como resultado 24.0. Al comparar el valor de $\mathrm{F}$ calculado con los valores tabulados a un nivel de significancia $a=0.01$, se encuentra que la $F$ observada es mayor que la $\mathrm{F}$ tabulada, $(24.00>5.34)$, por lo tanto, se rechaza la hipótesis nula y se acepta la hipótesis alternativa en el nivel de confianza de $99 \%$ (véase el cuadro 2).

Cuadro 2. Resumen de las fuentes de variación en los tratamientos (cantidades de suelo perdido).

\begin{tabular}{|c|c|c|c|c|c|c|}
\hline $\begin{array}{c}\text { Fuente de } \\
\text { variación }\end{array}$ & $\begin{array}{c}\text { Suma de } \\
\text { cuadrados }\end{array}$ & $\begin{array}{c}\text { Grados } \\
\text { de } \\
\text { libertad }\end{array}$ & $\begin{array}{c}\text { Varianza de } \\
\text { los cuadrados } \\
\text { medios }\end{array}$ & \multicolumn{2}{|c|}{$\begin{array}{c}\text { Valores tabulados de } \\
\text { la razón F }\end{array}$} & $\begin{array}{c}\text { F calculado } \\
\text { = CME/CMD }\end{array}$ \\
\hline $\begin{array}{c}\text { Entre tratamientos } \\
\text { (SCE) }\end{array}$ & $1,436.52$ & 2 & 478.84 & $a=0.05$ & $F=3.30$ & 24.00 \\
\hline $\begin{array}{c}\text { Dentro de } \\
\text { tratamientos (SCD) }\end{array}$ & 638.35 & 32 & 19.95 & $a=0.01$ & $F=5.34$ & \\
\hline Total (SCT) & $2,074.87$ & 34 & 59.29 & & & \\
\hline
\end{tabular}

Fuente: Elaboración propia.

Se concluye que existen diferencias estadísticas altamente significativas entre las medias de los tratamientos para rechazar la hipótesis nula, lo que indica que la variabilidad entre los tratamientos no se debe al azar sino a un efecto distinto entre dichos tratamientos. Posteriormente se realizaron pruebas de rango post ANOVA para determinar las diferencias particulares entre cada uno de los tratamientos (gráfico 2). Los resultados de la prueba de rango DHS de Tukey, con un nivel de confianza de $95 \%$ fueron 4.3, por consiguiente, una diferencia de por lo menos 4.3 Ton/ha entre cualquier par de medias resulta estadísticamente significativa.

Gráfico 2. Diagrama de árbol de las diferencias entre medias para las comparaciones de la prueba de rango DHS de las cantidades de suelo perdido.

Medias grupales

Diferencias

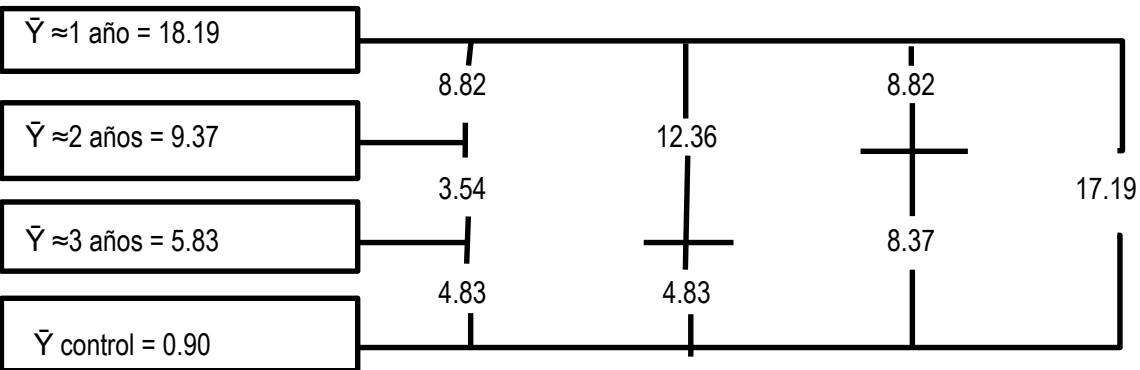

Fuente: Elaboración propia. 


\section{Variaciones en la pérdida de nutrientes}

La cuantificación de la pérdida de nutrientes del suelo requiere el análisis las pérdidas por diferentes vías: a) por escorrentía superficial, que se encuentran en forma soluble en las muestras de agua de escorrentía; b) por arrastre de suelo (erosión), en las muestras de sedimentos recogidos, que se encuentran en suspensión en los recipientes de recolección; c) por lixiviación, mediante muestreos de la solución del suelo, de materiales que se encuentran en solución en el suelo, y; d) por exportación por los cultivos. Para la determinación de las variaciones en el tiempo se requiere el muestreo y análisis en diferentes puntos dentro de un período de evaluación.

Por razones de disponibilidad de recursos para la ejecución de la investigación, solamente se realizaron muestreos de las parcelas a $25 \mathrm{~cm}$ de profundidad, en tres diferentes fechas, partiendo con el inicio, luego a mediados y al final del período de lluvias.

Por las razones mencionadas, no se espera realizar en este estudio la cuantificación de las variaciones en la pérdida de nutrientes relacionada con los diferentes procesos hídricos en el suelo. Solamente se realizan comparaciones de las cantidades encontradas en tres muestreos, sin pretender atribuir a la erosión de los suelos los cambios que se presenten en las parcelas analizadas.

\section{Asociación entre los diferentes tratamientos}

La asociación entre la precipitación y la escorrentía se analizó por medio del coeficiente de correlación lineal $r$ de Pearson y la regresión lineal. El coeficiente de correlación obtenido fue de 0.4533 , mostrando que existe una asociación positiva medianamente estrecha entre la precipitación y la escorrentía. Los valores tabulados mostraron un coeficiente de correlación r de 0.4182 para un nivel de significancia de 0.01 , por lo tanto, se rechaza la hipótesis nula para ese nivel de significancia. El coeficiente de regresión b (gráfico 3) obtenido es 0.0446. La pendiente de la recta, con una inclinación hacia arriba, indica una correlación positiva: un incremento de $1 \mathrm{~m} 3 / \mathrm{ha}$ de precipitación se relaciona con un incremento de $0.0446 \mathrm{~m} 3 / \mathrm{ha}$ en la escorrentía.

A manera de verificación de los niveles de significancia obtenidos se utilizó la prueba de razón $\mathrm{F}$ y la distribución muestral t aproximadamente normal, encontrándose la misma significancia para la asociación entre los factores. 


\section{Gráfico 3. Recta de regresión lineal entre los volúmenes de precipitación y los volúmenes de escorrentía.}

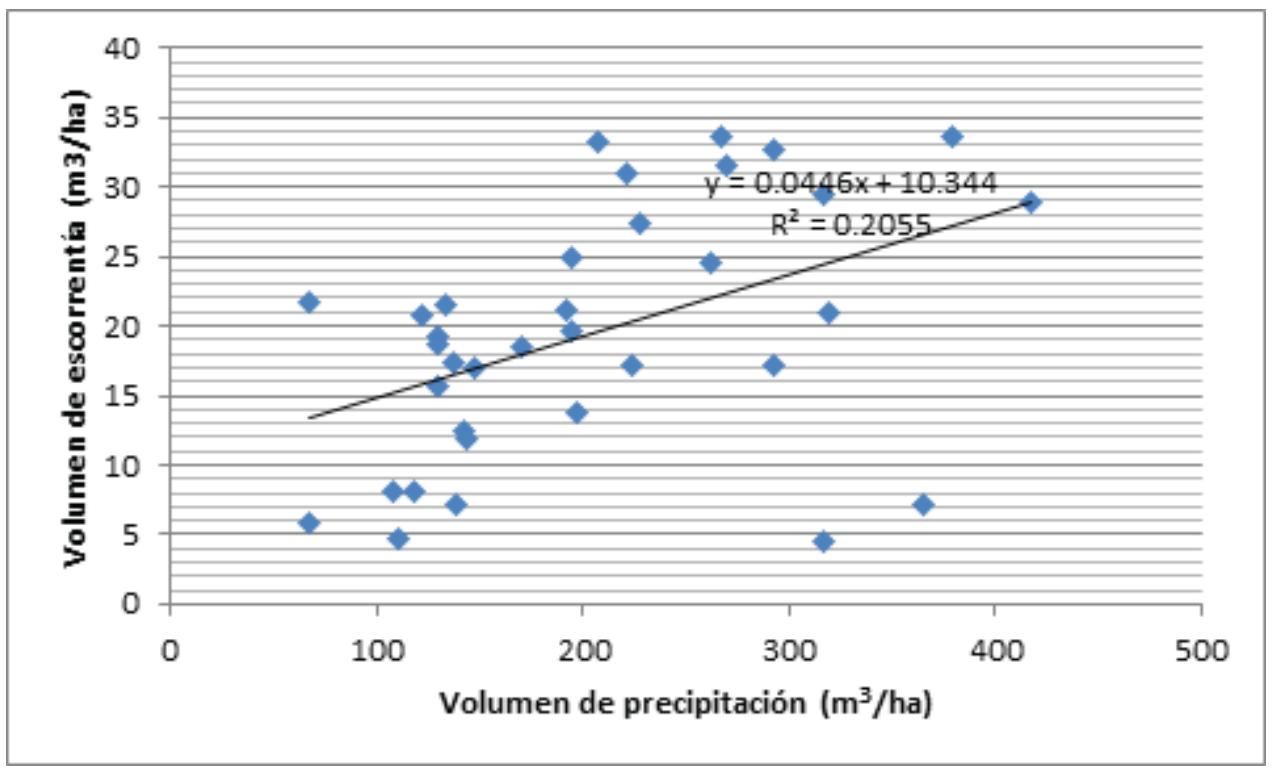

Fuente: Elaboración propia.

El coeficiente de correlación $r$ de Pearson obtenido entre el suelo erosionado y la escorrentía fue 0.6072 , indicando una asociación positiva medianamente estrecha entre los factores. Los cambios en los volúmenes de escorrentía se manifiestan en cambios en las cantidades de suelo perdido, no obstante que la coparticipación de otras variables inherentes a los suelos (textura, estructura, pendiente, capacidad de infiltración, permeabilidad, porosidad) y externas (intensidad de la lluvia, cobertura del suelo, prácticas de cultivo), influyen también en la erosión del suelo. Los valores tabulados del coeficiente de correlación, muestran un coeficiente de 0.5494 para un nivel de significancia de 0.10 , por lo tanto, se rechaza la hipótesis nula para ese nivel de significancia.

Sin embargo, aunque las correlaciones sean significativas, es necesario ser cuidadoso al afirmar que una fluctuación de la precipitación causa una fluctuación en la escorrentía y que una fluctuación de ésta causa una fluctuación en la pérdida de suelo. Si bien es cierto que estadísticamente existe una asociación explicada por los efectos que los volúmenes de precipitación ejercen para provocar la ocurrencia de la escorrentía y los efectos que los volúmenes de escorrentía pueden ejercer para provocar la ocurrencia de la erosión, a su vez, éstas pueden ser generadas por otros factores 
cuya participación puede representar importantes proporciones en la expresión de los fenómenos. Otras variables que no se han considerado en los cálculos, como la intensidad de las lluvias, las características propias de los suelos, el tiempo de los eventos, etc., pueden también ser responsables de aportar considerablemente a los volúmenes de escorrentía y a la pérdida de suelo.

El coeficiente de regresión b (gráfico 4) obtenido es 0.3695 . La pendiente de la recta muestra una inclinación hacia arriba, lo que indica una correlación positiva: un incremento de $1 \mathrm{~m} 3 / \mathrm{ha}$ de precipitación se relaciona con un incremento de 0.3695 ton/ha (369 Kg/hectárea) en la pérdida de suelo.

A fin de verificar los resultados de los niveles de significancia obtenidos se utilizó la prueba de razón $\mathrm{F}$ y la distribución muestral t aproximadamente normal, encontrándose la misma significancia para la asociación entre los factores.

Gráfico 4. Recta de regresión lineal de las cantidades de erosión del suelo y los volúmenes de escorrentía.

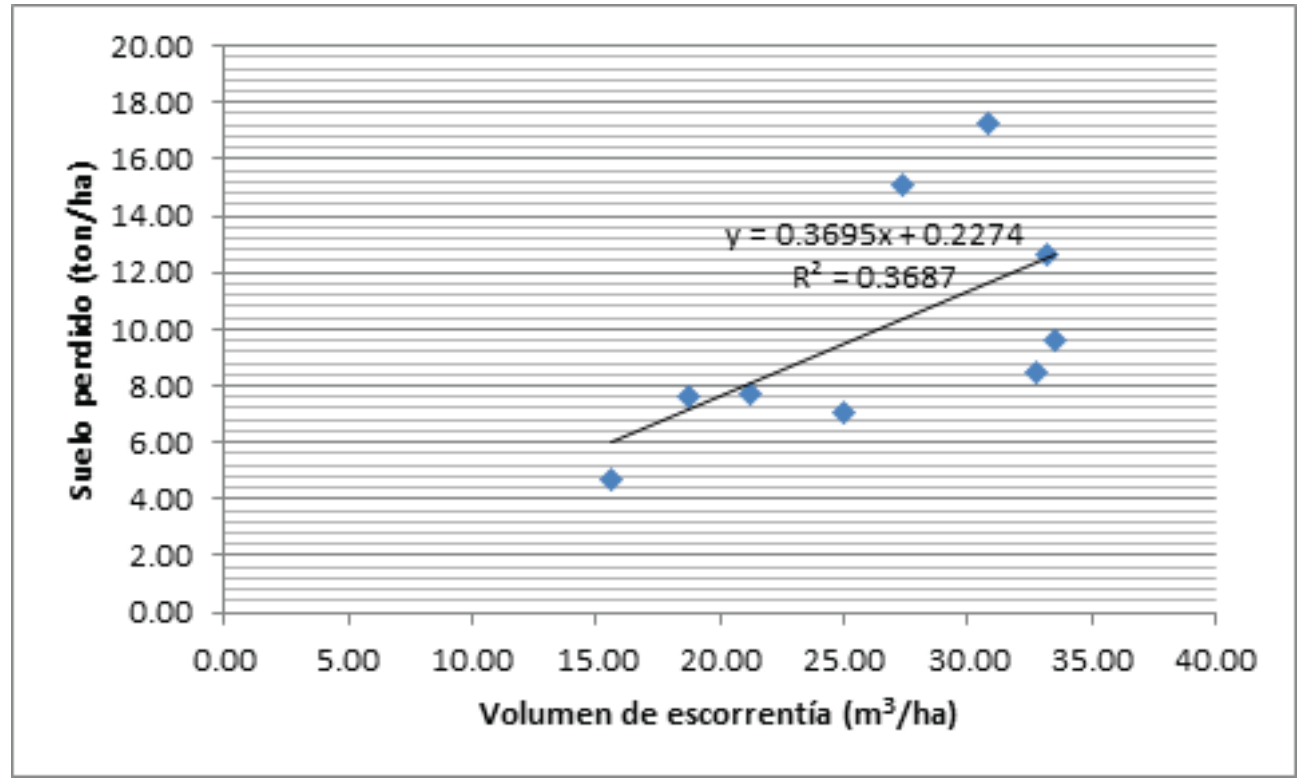

Fuente: Elaboración propia. 


\section{DISCUSIÓN}

La reducción del escurrimiento del agua a medida que se va incrementando la edad en la plantación de café en el proceso de crecimiento se explica (en un 39\%) por el desarrollo de la cubierta foliar protectora que intercepta el agua precipitada; sin embargo, otros factores coparticipan en la ocurrencia del fenómeno, explicando el porcentaje restante, como las diferencias en el microrrelieve de los terrenos, las pendientes, la profundidad de los suelos, las variaciones e intensidad en las precipitaciones, entre otros. Las variaciones en las cantidades de suelo perdido entre los tratamientos son explicadas por los cambios en la cobertura vegetal (en un 66\%); pero también por otros factores que participan en una proporción considerable en la ocurrencia del fenómeno.

Tanto el escurrimiento como la erosión son considerablemente menores en las parcelas de bosque en las cuales se presenta una mayor cobertura foliar, además de una mayor cantidad de material orgánico que absorbe el agua depositada sobre la superficie e impide el arrastre de las partículas. Aunque el coeficiente de correlación muestra una relación medianamente estrecha entre la precipitación, la escorrentía, y entre ésta la pérdida de suelo, extrapolar los resultados a otras zonas similares debe ser una acción que se haga con cuidado, considerando la intervención de diferentes variables que hacen que la relación sea imperfecta.

La metodología es eficaz para la finalidad del estudio, relacionada con la demostración de hechos conocidos, como, mostrar que la escorrentía y la erosión son mucho menores en una parcela cubierta de vegetación que en una parcela desnuda, y mostrar argumentos sobre cómo la cubierta vegetal influye en el control de la erosión. Los resultados deben interpretarse con las debidas reservas, dadas las características del fenómeno en el que participan diferentes variables, cuya acción es difícil de aislar en una investigación ejecutada en condiciones de campo. La obtención de resultados más consistentes requeriría, además, la ejecución de observaciones en series cronológicas en el mediano o largo plazo, para analizar la influencia de la precipitación sobre la escorrentía y de ésta sobre la erosión y sobre la pérdida de nutrientes, y realizar inferencias con respecto al fenómeno.

Las estimaciones de las pérdidas de suelo en sistemas de producción de café presentan bastante variabilidad entre diferentes estudios. Hincapié y Ramírez (2010) estiman que la pérdida de suelo por erosión en la zona cafetalera colombiana se encuentra en 6 a 30 ton/ha/año, mientras que Chávez y Bojórquez (2012) la valoran 
en 19 a 34 ton/ha/año para una zona cafetalera en Ahuachapán, El Salvador. Entretanto, Martínez, López y López (2001) señalan rangos de entre 0.047 y 1.37 ton/ha para café en zonas de laderas en Oaxaca, México y Bermúdez (1980) señala cantidades de 0.366 ton/ha en parcelas de café en Turrialba, Costa Rica, pero Quiñonez (2012) indica pérdidas hasta de 48 ton/ha para una microcuenca en Zacapa, Guatemala. En la presente investigación se estimaron en promedio, pérdidas de 25 ton/ha en los lotes cultivados con café.

Las variaciones se manifiestan en función de diferentes factores relacionados con el clima, el relieve, el suelo y con el sistema de producción. Sin embargo, en investigaciones a nivel de campo, resulta difícil separar y controlar los diferentes factores. La utilización de diferentes métodos directos e indirectos (PASOLAC, 2005; Hudson, 1997) podría ser fuente considerable de variación en los resultados.

\section{CONCLUSIONES}

1. Se encontraron diferencias estadísticas que indican que la reducción de la escorrentía entre las edades no se debe al azar sino a los cambios en la densidad del follaje del cultivo de café que se van desarrollando a medida que éste va creciendo, generando una cubierta vegetal protectora ante la precipitación.

2. El volumen de escorrentía no es significativamente diferente entre cada una de las edades en forma sucesiva, probablemente por ser un período relativamente corto para que el follaje desarrollado produzca la suficiente cobertura para reducir de forma significativa los volúmenes de escorrentía.

3. Se encontraron diferencias en las pérdidas de suelo entre cada uno de los tratamientos, en las cuales puede observarse una reducción de manera proporcional de las cantidades de suelo perdido a medida que se va incrementando la edad del cultivo. La cantidad de suelo perdido es significativamente diferente entre el tratamiento de $\approx 1$ y el de $\approx 2$ años y entre el tratamiento de $\approx 3$ años y el control. El incipiente desarrollo del sistema foliar el primer año permite las condiciones para la pérdida de grandes cantidades de suelo.

4. Las diferencias en la escorrentía y en las pérdidas por erosión entre la cobertura boscosa y las diferentes edades, demuestra la capacidad del follaje para interceptar y amortiguar la energía cinética de las gotas de lluvia que generan la escorrentía y el arrastre de las partículas de suelo, el potencial de absorción y almacenamiento de la capa de material orgánico que cubre el suelo y del tejido radicular para influir en la circulación del agua en el suelo. 
5. No puede establecerse con los análisis realizados, la existencia de variaciones en las cantidades de nutrientes producto de la pérdida física del suelo, ya que esto requeriría la evaluación de las pérdidas por escorrentía superficial, por arrastre de suelo, por lixiviación y por exportación en los cultivos, lo que se encuentra fuera de las posibilidades financieras del presente estudio. Se recomienda la ejecución de estudios, si es posible, longitudinales, en los que puedan analizarse cambios en las cantidades de nutrientes perdidos en el suelo, que puedan contribuir a determinar cómo afecta la erosión hídrica al crecimiento y la productividad del café.

6. Las correlaciones entre la precipitación y la escorrentía y entre ésta y la pérdida de suelo muestran que existe una asociación positiva media en ambos casos. Sin embargo, aunque estadísticamente existe una asociación explicada por los efectos que la precipitación puede ejercer para provocar la ocurrencia de la escorrentía, y por los efectos que la escorrentía ejerce sobre las cantidades de suelo perdido, la participación de otros factores puede representar importantes proporciones en la expresión del fenómeno, como la intensidad de las lluvias, las características propias de los suelos, el tiempo de los eventos, entre otros.

\section{AGRADECIMIENTOS}

Al Coordinador Regional de Investigación Científica del CUROC, Kevin Estévez, por todo el apoyo en la ejecución de la investigación. A la Dirección de Investigación Científica y Posgrados de la UNAH, por el apoyo para el desarrollo del estudio a través de la beca de investigación y la asesoría brindada.

\section{REFERENCIAS BIBLIOGRÁFICAS}

Bermúdez, M., (1980). Erosión hídrica y escorrentía superficial en el sistema de café, poró y laurel. Turrialba, Costa Rica: CATIE.

Chávez Ramírez, Krissia Marlyn y Bojorquez Aguirre, José Eduardo. (2012). Plan de conservación de suelos en laderas y fertilización para el cultivo de café en Ahuachapán, El Salvador. (Tesis de licenciatura). Escuela Agrícola Panamericana. Zamorano, Honduras.

Chela, E., Monar, C., Valverde, F. y Cruz, E. (2008). Evaluation of soil loss from water erosion in three production systems of the Alumbre River watershed, Ecuador. Quito: SANREM CRSP.

Cisneros, J., et al, (2012). Erosión hídrica: principios y técnicas de manejo. Río Cuarto - 
Argentina: UniRío Editora.

Consejo Intermunicipal Higuito, (2006). Indicadores socioeconómicos línea de base del Municipio de Dolores, Copán (en el marco de los ODM y la ERP). Dolores, Copán: Levantados y preparados con el apoyo técnico y financiero de la Agencia de Cooperación Internacional del Japón (JICA).

Da Silva, D., Vaquero, R. y Tejada, J. (2006). Propuesta metodológica para medir la escorrentía en sistemas agropecuarios del cantón de Guácimo, zona del caribe de Costa Rica. Tierra Tropical, 2 (2): 133-142.

De Alba, S., Benito, G., y Pérez, A., (2002). Parcelas experimentales para el estudio de la erosión hídrica: finca experimental la Higueruela. Cuadernos de la Sociedad Española de Ciencias Forestales, 13 (1): 45-53.

Felipe-Morales, C., (1996). Manual evaluaciones en la parcela de escorrentía y erosión: Método de Medición directa de la erosión del suelo. Lima: GTZ.

Garzón, H., Faustino, J., Ferreira, P. y Kass, D. (1993). Evaluación de la erosión hídrica y escorrentía superficial, bajo sistemas agroforestales, en tierras de laderas. Turrialba, Costa Rica: CATIE.

Hayward, J., (1968). The measurement of soil loss from fractional acre plots. Lincoln Papers in Water Resources. Canterbury, New Zealand: The New Zealand Agricultural Engineering Institute, Lincoln College.

Hincapié, E., Salazar, L. (2011). Impacto de la erosión sobre las propiedades físicas y químicas del suelo y la producción de café. Revista Cenicafé, 62 (2): 79-89.

Hincapié, E. Ramírez, F. (2010). Riesgo a la erosión en suelos de ladera de la zona cafetera: avances técnicos CENICAFE. Chinchiná, Caldas, Colombia.

Hudson, N. (1997). Medición sobre el terreno de la erosión del suelo y de la escorrentía. (Boletín de Suelos de la FAO - 68). Roma: FAO.

Konz, N., Baenninger, D., Konz, M., Nearing, M. y Alewell, C. (2010). Process identification of soil erosion in steep mountain regions. Hydrology and Earth System Sciences, 14 (1): 675-686.

Little, T. y Hills, F., (1989). Métodos estadísticos para la investigación en la agricultura $\left(2^{\mathrm{a}}\right.$ ed.). México: Trillas.

Martínez, M., López, R. y López, C. (2001). Caracterización geográfica y escalamiento de cuencas en zonas de laderas de Oaxaca. Guanajuato, Guanajuato, México: XI Congreso Nacional de Irrigación.

Lobos, I. (Ed.), (2013). Tierra y suelos en los Objetivos de Desarrollo Sostenible Rio+20, Estudio de caso Guatemala. Instituto de Estudios Avanzados de Sostenibilidad.

Pennock, D. (2004). Designing field studies in soil science. Canadian Journal of Soil Science, 84 (1): 1-10.

Perret, S. (1993). Una metodología simple para el dimensionamiento de parcelas de escorrentía. Ciencia e Investigación Forestal, 7 (2): 339-357.

Programa para la Agricultura Sostenible en Laderas de América Central (PASOLAC), (2005). Manual de métodos sencillos para estimar erosión hídrica. Managua, Nicaragua: UNA, PASOLAC, CIAT. 
Quiñonez Berganza, Gerardo Osvely. (2012). La degradación del suelo por erosión hídrica en cultivos de granos básicos y café en la microcuenca Torjá, cuenca del río Grande de Zacapa, Guatemala. (Tesis de maestría). Centro Agronómico Tropical de Investigación y Enseñanza. Turrialba, Costa Rica.

Santos Velásquez, Alfredo Alberto. (2010). Determinación de la erosión hídrica para diferentes rangos de pendientes en finca Santa Albina, municipio de Colomba Costa Cuca, departamento de Quetzaltenango. (Tesis de licenciatura). Universidad Rafael Landívar. Guatemala.

Secretaría de Recursos Naturales y Ambiente (SERNA) (2010). Estrategia Nacional de Cambio Climático Honduras. Tegucigalpa, Honduras: Dirección Nacional de Cambio Climático.

Vega Torres, Leonardo Patricio. (2008). Evaluación de la erosión hídrica superficial por parcelas experimentales en suelos desnudos de la región de Coquimbo. (Tesis de licenciatura). Universidad de Talca. Chile.

Zamora, C. (2011). Crisis rural, cambio climático y pobreza: Hacia la búsqueda de alternativas para la definición de políticas públicas en México. México, D.F.: Oxfam México. 